\title{
Editorial: The 10 year's highlights and future developments in Neuro-oncology: 10th Anniversary of Neuro-oncological Update, Salzburg, 29th January 2010
}

\author{
H. Kostron \\ Department of Neurosurgery, Medical University Innsbruck, Innsbruck, Austria
}

In January 2010 the 10th anniversary of the neuro-oncological update was celebrated. For this anniversary the topic was chosen accordingly: “The 10 year's highlights and future developments in malignant brain tumours".

The faculty featured highly acknowledged experts from Slovenia, Germany, Holland and Austria. This symposium demonstrated that the greatest achievement of the last 10 years was the close and friendly interaction and cooperation with all disciplines dealing with neuro-oncology ranging from neuropathology, molecular diagnostics, neurology, neurosurgery, radio-oncology and haemato-oncology and also psycho-oncology. The greatest impact on therapeutic decision-making had the characterisation of the molecular signature of the tumour and therapeutically certainly Temozolomide, which created the new enthusiasm in neuro-oncology. The most recently introduced anti-VEGF therapy in combination with Temozolomide will be a further add-on armentarium in the treatment of malignant gliomas. Patients' care and acceptance - thus the improvement in quality of life - has gained significant importance.

The Update started with the presentation of J. Hainfelllner, Vienna, about the interaction of neuropathology and neurooncology, the translational research and its progress in the management and therapy in high-grade gliomas. The important message was that the evaluation of the molecular markers e.g. MGMT status is strongly depended on the methods employed. U. Bogdahn, Regensburg, covered the various concepts of CHT and also outlined the importance of new approaches by stem cells and vaccination. The state of the art of advanced MR imaging was impressively presented by K. Nussbaumer (Linz). This included also new techniques to differentiate between real and pseudo tumour progression and 3D functional imaging. Neurosurgical highlights were presented by V. V. Dolenc (Lubliana). He demonstrated that the combination of new imaging techniques combined with neuro-anatomical knowledge and microsurgical skills are almost without limits in the surgery of brain pathologies. The neurosurgical procedure as first step of diagnosis and the importance of maximal safe cyto-reduction was clearly demonstrated. The second mainstay after surgery remains radiotherapy. This update was presented by K. Dieckmann, Vienna with the focus on re-radiation

Correspondence: Herwig Kostron, Department of Neurosurgery, Medical University Innsbruck, Anichstrasse 35, 6020 Innsbruck, Austria.

E-mail: herwig.kostron@i-med.ac.at of malignant tumours. John Wolbers, Neurosurgeon from Rotterdam, reported on the change of paradigm induced by radio surgery. Twenty-five percent of patients over 60 years old will not return to home due to high morbidity after surgery. Contrastingly, radio surgery provides 10-year progression free survival in over $90 \%$ without major, invalidating, complications. Furthermore, in symptomatic, large tumours the availability of radio surgery does allow surgeons to be more conservative in their approach. These practices lead to an ongoing shift from tumour removal towards tumour control by radio surgery.

The third mainstay in neuro-oncological treatment is chemotherapy. C. Marosi, Vienna, covered this wide topic. The image of neuro-oncology has changed from the exotic entity to a model for dynamic interdisciplinary research, which has occurred within the last ten years and is tightly related with Temozolomide (TMZ). Modern day development of neuro-oncology would not have been possible without Temozolomide.

The final presentation of the invited speakers was given by E. Sizoo, Amsterdam, covering the symptom control and quality of life in the end of life phase of brain tumour patients. Symptom burden in the end-of-life phase of high-grade glioma patients was high. This contribution showed the advances in the management of patients in the end of life phase, which changed significantly over the past 10 years.

The case reports were presented covering various pathologies and experimental protocols. One outstanding approach was reported by D. Putzer et al., Innsbruck, reporting upon a long-lasting survival of a glioblastoma multiforme after local intracavitary targeted therapy with a radio peptide (90Y-DOTA-TOC). This part of the symposium is very much favoured by the participants because of the interactive and lively discussion. At the end the invited speakers as well as the audience gave short comments to the development of neurooncology for the next 10 years. Surgery will remain as mainstay for diagnosis and decompression followed by radiotherapy for initial sterilisation of the tumour bed and surrounding tissue. However since gliomas are a disease of the brain, chemotherapy will play the final role to have permanent control whether by means of cytotoxic action or remodulation of malignant cells. All the participants were grateful for the organisation and support of AESCA/MSD Austria.

Conflict of interest

The authors declare that there is no conflict of interest. 